Original Article

\title{
GREEN BIOSYNTHESIS OF MAGNETIC IRON OXIDE NANOPARTICLES OF VITEX NEGUNDO AQUEOUS EXTRACT
}

\author{
P. KARNAN, A. ANBARASU, N. DEEPA, R. USHA \\ Department of Zoology, Presidency College, Chennai 600005 \\ Email: johnjalu@gmail.com
}

Received: 20 Jan 2018, Revised and Accepted: 08 Apr 2018

\section{ABSTRACT}

Objective: The green synthesis of magnetic iron oxide nanoparticles is a convenient, economical, rapid and eco-friendly method compared to physical and chemical synthesis methods.

Methods: In the present study iron oxide nanoparticles synthesized by Vitex negundo leaves extract.

Results: The formation of iron oxide nanoparticles was confirmed by the colour change and further characterized by UV-Visible Spectroscopy and XRD. The morphology and the size of nanoparticles were analyzed by SEM and HR-TEM analysis.

Conclusion: On the basis of this research work, green synthesized iron oxide nanoparticles can be a good source for alternative therapy for human diseases.

Keywords: Green synthesis, Iron oxide, Nanoparticles, Vitex negundo

(C) 2018 The Authors. Published by Innovare Academic Sciences Pvt Ltd. This is an open access article under the CC BY license (http://creativecommons.org/licenses/by/4.0/) DOI: http://dx.doi.org/10.22159/ijcpr.2018v10i3.27220

\section{INTRODUCTION}

In nanotechnology, magnetic iron oxide nanoparticles are microscopic particles and sized between 1 and 100 nanometers [1] It has unique and most important property i. e larger surface area than superior particles which cause them to be more reactive to some other molecules. They are extensively synthesized by using physical and chemical methods. These synthesized methods are needed to use high energy, temperature, toxic chemicals and expensive. The primary goal of nanotechnology is to develop convenient, economical, rapid and eco-friendly green synthesis methods [2]. Magnetic iron oxide nanoparticles research is presently an area of passionate scientific interest due to a broad range of prospective applications. It is used as catalysis [3] high-density magnetic storage media [4] and chemical sensors [5]. It is an effective nano-agent to remove a number of pollutants from water resources [6]. It has many important biomedical applications such as for targeted drug delivery in clinical trials [7], contrast agents in magnetic resonance imaging (MRI) [8], antibacterial activity [9] and anticancer activities [10]

Vitex negundo Linn. is a large aromatic shrub distributed throughout India. Herbal medicine, rather than merely curing a particular disease, aims at returning the body back to its natural state of health. It has been used since ancient times as a female remedy and also for pains in Ayurveda and also in Roman medicine. It became known as the chaste berry tree. This species is globally distributed in Indo-Malesia, cultivated in America, Europe, Asia and West Indies. Within India, it is found throughout the greater part of India, in the outer Himalayas. Myriad medicinal properties have been ascribed to Vitex negundo Linn. and the plant has also been extensively used in the treatment of a plethora of ailments as traditional medicine, folk medicine and pharmacological evidence. Traditionally the leaves of Vitex negundo Linn. are documented to possess antibacterial, antitumor, astringent, febrifuge, sedative, tonic and vermifuge. It has been reported to possess potent pharmacological properties like anti-inflammatory, anti-rheumatic, antibiotic, Hepatoprotective, antioxidant, anticonvulsant, oxidative stress, anti-androgen, snake venom neutralization and anti-allergic activities. The various chemical constituents like flavonoids, flavones glycosides, volatile oil, triterpenes, tannins and many others were identified in this plant. This review gives a bird's eye view mainly on the pharmacokinetic characteristics. traditional uses, phytochemistry and pharmacological actions of Vitex negundo Linn. Furthermore, a study by Lv et al. (2013) [11] revealed sesquiterpenes are the main constituents in the essential oil of Vitex negundo. Considering the ethnomedicinal properties and reported activities of Vitex negundo L., in the present investigation was preferred for nanoparticles synthesis.

\section{MATERIALS AND METHODS}

\section{Collection and identification of plant}

Fresh healthy leaves of Vitex negundo were collected from Presidency college, Chennai and were authentically identified by Prof. P. Jayaraman, Institute of Herbal Science, Plant Anatomy Research Centre, West Tambaram, Chennai, India as Verbenanceae with voucher specimen number PARC/2017/3149.

\section{Synthesis of iron oxide nanoparticles using Vitex negundo extract}

About $200 \mathrm{~g}$ of fresh healthy leaves of Vitex negundo were washed thoroughly with running tap water and double distilled water, cut into fine pieces and shade dried for $15 \mathrm{~d}$ under dark condition. After drying the leaves were powdered using a kitchen blender. The powdered leaves were soaked in the $400 \mathrm{ml}$ of double distilled water for overnight in a fridge for $4{ }^{\circ} \mathrm{C}$ and then the rinsed mixtures were boiled for $10 \mathrm{~min}$. The extracts were cooled to room temperature and then filtered through Whatman filter paper. Iron oxide nanoparticles were synthesized by taking $\mathrm{FeCl}_{3} \cdot 6 \mathrm{H}_{2} \mathrm{O}$ and $\mathrm{FeCl}_{2} .4 \mathrm{H}_{2} \mathrm{O}$ (1:2 molar ratios) and were dissolved in $100 \mathrm{ml}$ of double distilled water in a $500 \mathrm{ml}$ beaker and heated at $80{ }^{\circ} \mathrm{C}$ with mild stirring using magnetic stirrer under atmospheric pressure. After $10 \mathrm{~min}, 20 \mathrm{ml}$ of the aqueous solutions of Vitex negundo extract was added to the mixture, immediately the light green colour of the Vitex negundo extract of the mixture changed to dark brownish colour. After $10 \mathrm{~min}, 20 \mathrm{ml}$ aqueous solution of sodium hydroxide was added to the mixtures with the rate of $3 \mathrm{ml}$ per minutes for allowing the iron oxide precipitations uniformly. From the first addition of sodium hydroxide, the dark brown mixture changed to black suspended particles. The mixture was allowed to cool down to room temperature and the iron oxide nanoparticles were obtained by decantation to form magnetite. The magnetites formed were washed 3 times with double distilled water and 3 times with ethanol and air dried at room temperature. 


\section{Characterization}

The surface Plasmon resonances (SPR) of synthesized iron oxide nanoparticles have been studied by UV-Vis double-beam spectrophotometer Elico-Bl-198 using the software Spectral Treats Version 2.37.4 Rel-1 in the range of 300 to $700 \mathrm{~nm}$. The diffraction pattern was recorded by Seifert Rayflex Software which provides control modules for the complete range of diffractometer accessories together with the corresponding analysis software XRD with $\mathrm{Cu}-\mathrm{K} \alpha$ radiation. Morphological analysis of nanoparticles was done using Vega 3 Tescan SEM machine. The morphology of magnetic iron oxide nanoparticles was viewed under a Transmission electron microscope (HR-TEM, Jeol model 3010, at $200 \mathrm{Kv}$ and 104.1 $\mu \mathrm{A})$.

\section{RESULTS AND DISCUSSION}

\section{Synthesis of Iron oxide nanoparticles}

Fig. 1 shows the iron oxide nanoparticles synthesized by chemical precipitation method. The powder form of synthesized nanoparticles using the aqueous extracts of Vitex negundo black in colour (fig. 1) and the intensity of color increased with time and dosage of plant extract it indicates the more growth of nanoparticles. The color change is the most easy and commonly used indication of the metal nanoparticles formation. The plant extract contained much organic content. Hence the mechanism study of iron oxide nanoparticles formation is a little difficult. However, the organic compound, which is present in the plant extracts act as a reducing as well as capping or binding agent to form iron oxide nanoparticles.

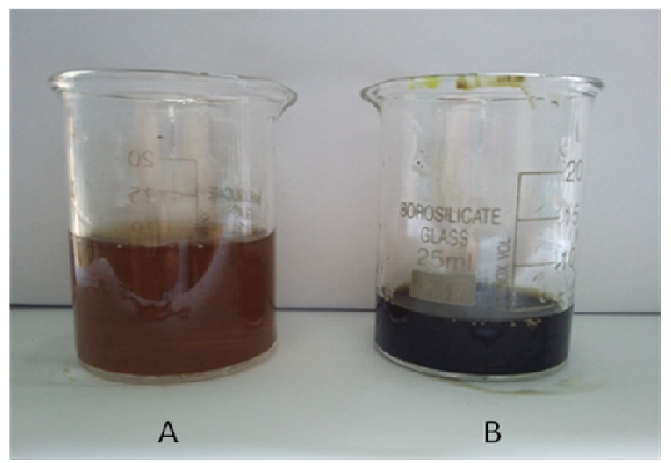

Fig. 1: A-Vitex negundo leaves aqueous extract, B-Vitex negundo leaves aqueous extract based synthesized iron oxide nanoparticles

\section{Characterization of Iron oxide nanoparticles}

\section{UV-visible spectroscopy}

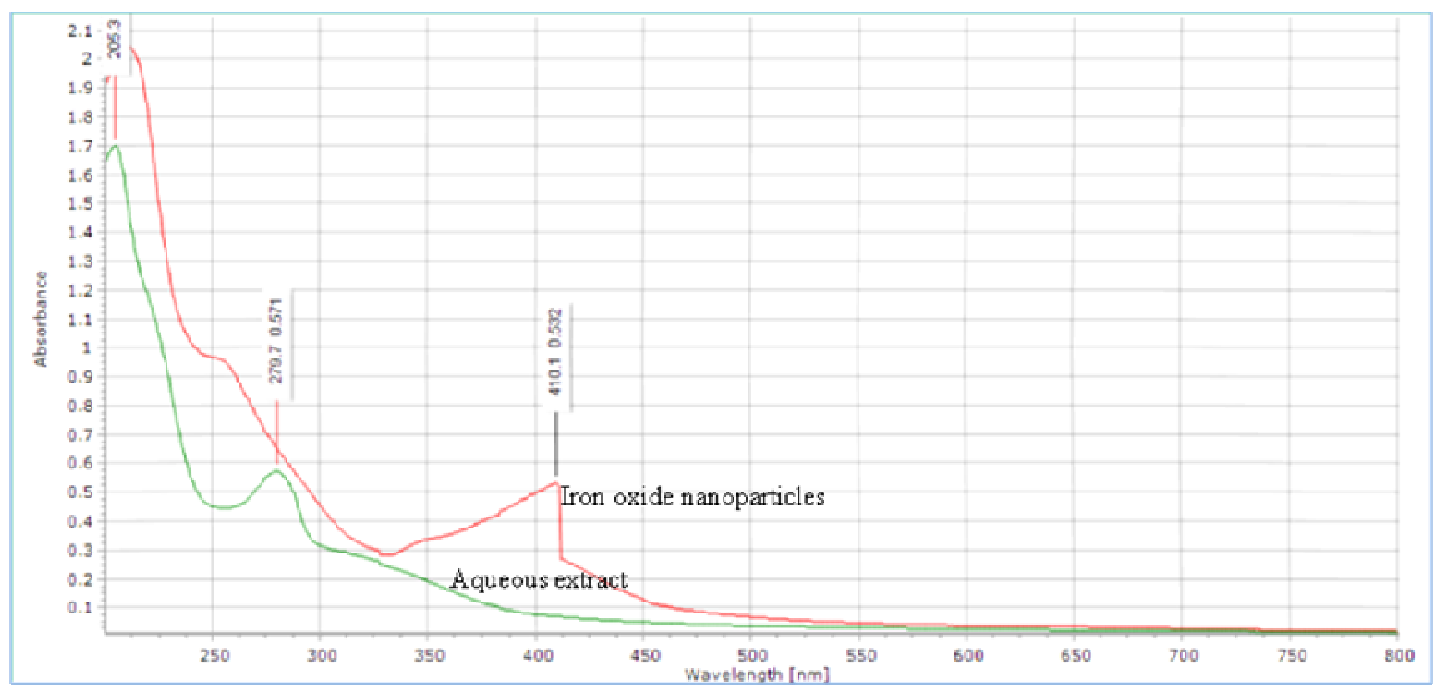

Fig. 2: Shows the UV-Visible spectra of Vitex negundo leaves iron oxide nanoparticles of with the varing $\lambda$ max peaks around at $410 \mathrm{~nm}$ respectively

\section{XRD spectral analysis}

Fig. 3 shows the XRD pattern of Iron oxide nanoparticle. The narrow peaks in the fig. confirm the crystalline structure of iron oxide nanoparticles. The Structural characterization of the material was performed by using Powder X-Ray diffraction (XRD) analysis. The sample showed the major characteristic peaks for prepared crystalline metallic nanoparticles at two data values of $24.2,33.1,35.7,40.9,49.4$ 54.1, 57.6, 62.6, and 64.0degrees corresponding to (012), (104), (110), (113), (024),(116), (018), (214), and (300) respectively. The XRD analysis of Vitex negundo iron oxide nanoparticles clearly indicates the presence of high crystalline phases while the " $\mathrm{d}$ " value point out the presence of $\mathrm{Fe}_{2} \mathrm{O}_{4}$ and ${ }^{\gamma}$-Fe2O3. The $\mathrm{d}$ values are calculated from standard XRD pattern for Fe304 refers to the JCPDS data (88-2334). The plasma synthesis permits to produce highly crystalline nanoparticles in which the degree of the disorder can be tuned. The transmission electron microscopy analysis points out that, increasing the plasma current, the morphology of the nanoparticles changes from a symmetric spherical shape to a lower symmetry hexagonal shape as shown in the fig. 3.

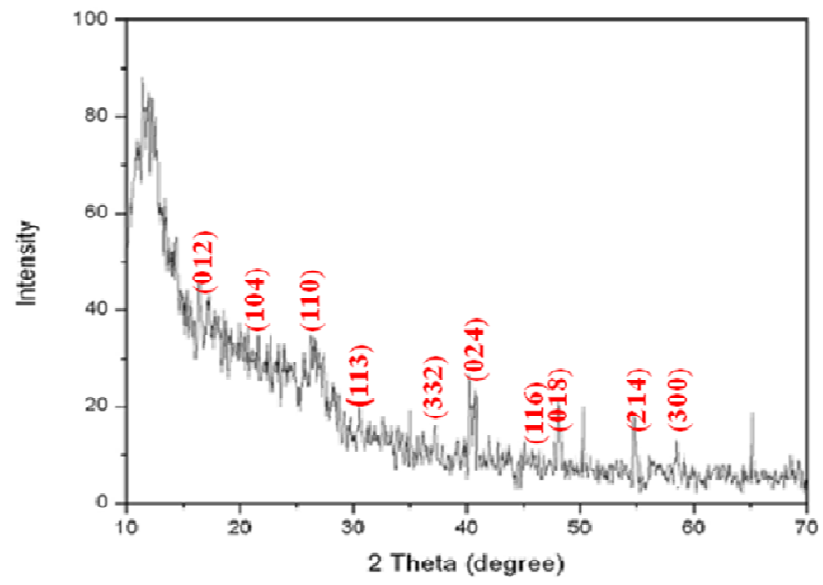

Fig. 3: XRD analysis of Vitex negundo iron oxide nanoparticles 


\section{SEM analysis}

Fig. 4 shows the SEM image of Iron oxide nanoparticle synthesized by chemical precipitation method and the size of the particles in iron oxide nanoparticles were found to have agglomerated in its structures showing spherical, oval and triangle shapes. This is comparable to the findings of Wang et al. (2014) [12] who reported the size of iron nanoparticles by using Eucalyptus leaves was diameter ranging from 20 to $80 \mathrm{~nm}$. On the contrary, Latha and Gowri (2014) [13] analysed the SEM image of iron oxide nanoparticles synthesized by Carica papaya leaf extracts demonstrated uniformly distributed spherical shaped particles. The increase in the size of nanoparticles confirms the presence of iron oxide nanoparticles with agglomerated in its structures.

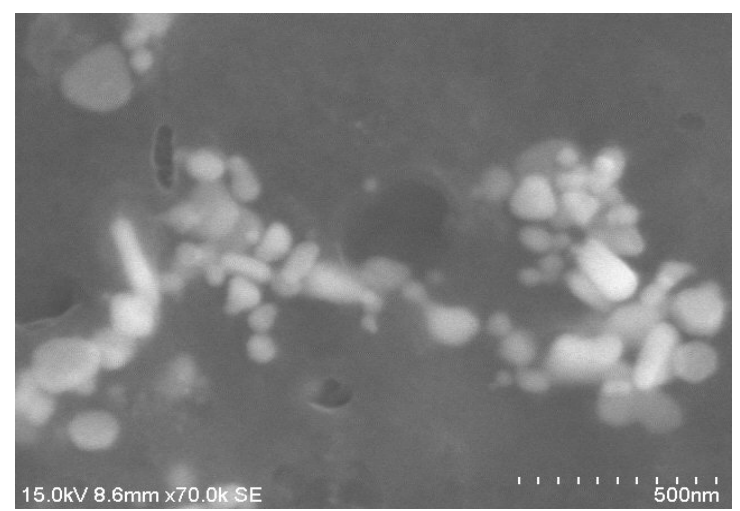

Fig. 4: FE-SEM image of Vitex negundo iron oxide nanoparticles

\section{HR-TEM analysis}

In addition, the iron oxide nanoparticles were analyzed by HRTransmission Electron Microscopy. Fig. 5 shows the HR-TEM image of Vitex negundo iron oxide nanoparticles prepared from aqueous leaves extract of TEM image reveal that the iron oxide nanoparticles have the average core diameter of 20 to $22 \mathrm{~nm}$ and the nanoparticles are agglomerated and cluster. The aggregation may be due to a magnetic property of Iron oxide nanoparticles as shown in the fig. 5 Iron oxide nanoparticles have a large surface to volume ratio and therefore possess high surface energies and consequently, they tend to aggregate so as to minimize the surface energies. The SAED pattern (fig. 6) reveals that nanoparticles are polydispersive in nature. HR-TEM image also revealed the successful synthesis of nanosized iron oxide particles, the average core diameter of $100 \mathrm{~nm}$ and the nanoparticles are agglomerated and cluster. The aggregation might be due to a magnetic property of Iron oxide nanoparticles. Iron oxide nanoparticles have a large surface to volume ratio and possess high surface energies. Accordingly, they tend to aggregate so as to minimize the surface energies [14].

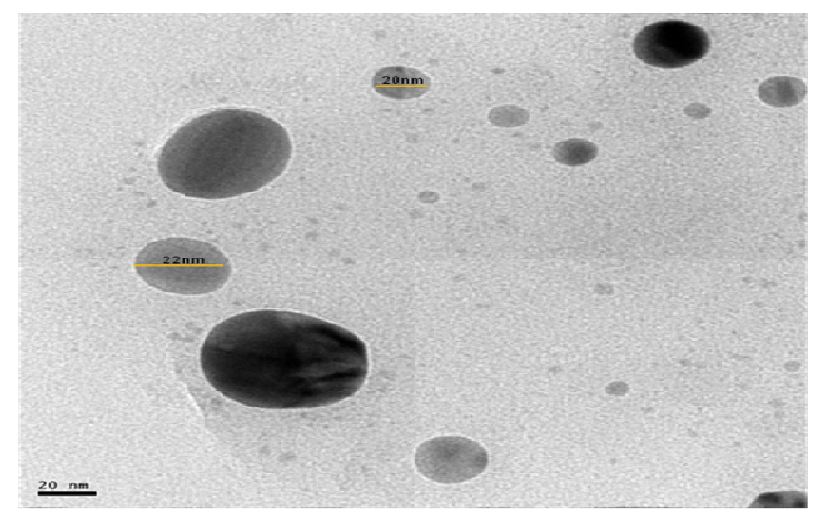

Fig. 5: HR-TEM image of Vitex negundo iron oxide nanoparticles

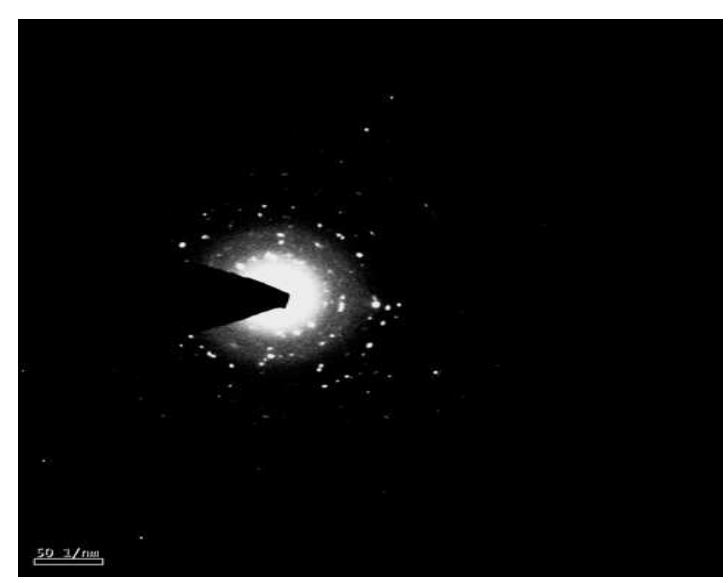

Fig. 6: SAED pattern image of Vitex negundo iron oxide nanoparticles

\section{CONCLUSION}

For the first time, biosynthesis of magnetic iron oxide nanoparticles by using Vitex negundo aqueous extract is reported. Measurement of UV, IR, XRD, SEM, and HR-TEM analysis confirmed the structures. On the basis of this research work, green synthesized iron oxide nanoparticles can be a good source for alternative therapy for human diseases. The study can be extended for nanomedicine application and preclinical studies in relevant animal models.

\section{AUTHORS CONTRIBUTIONS}

All the author have contributed equally

\section{CONFLICT OF INTERESTS}

Declared none

\section{REFERENCES}

1. Thakkar KN, Mhatre SS, Parikh RY. Biological synthesis of metallic nanoparticles. Nanomedicine 2010;6:257-62.

2. Allouche J. Synthesis of organic and bioorganic nanoparticles: an overview of the preparation methods. Springer London. In: Nanomaterials A Danger or a Promise; 2013. p. 27-74.

3. Weiss W, Ranke W. Surface chemistry and catalysis on welldefined epitaxial iron-oxide layers. Prog Surf Sci 2002;70:1-15.

4. Zeng H, Li J, Liu JP, Wang ZP, Sun SH. Exchanged-coupled nanocomposite magnets via nanoparticle self-assembly. Nature 2002;420:395.

5. Raj VB, Nimal AT, Parmar Y, Sharma MU, Sreenivas K, Gupta V. Cross-sensitivity and selectivity studies on $\mathrm{ZnO}$ surface acoustic wave ammonia sensor. Sens Actuators B 2010;147:517.

6. Feng J, Hu X, Yue PL, Zhu HY, Lu GQ. Degradation of azo-dye orange II by a photoassisted fenton reaction using a novel composite of iron oxide and silicate nanoparticles as a catalyst. Ind Eng Chem Res 2003;42:2058-66.

7. Kim DK, Zhang Y, Voit W, Rao KV, Kehr J, Bjelke B, et al. Superparamagnetic iron oxide nanoparticles for biomedical applications. Scripta Materialia 2001;44:1713-7.

8. Modo MMJ, Bulte JWM. Molecular and cellular MR imaging; CRC Press: Boca Raton, FL; 2007.

9. Ismail RA, Sulaiman GM, Abdulrahman SA, Marzoog TR Antibacterial activity of magnetic iron oxide nanoparticles synthesized by laser ablation in liquid. Mater Sci Eng Carbon 2015;53:286-97.

10. Nagajyothi PC, Pandurangan M, Kim DH, Sreekanth TVM, Shim J. Green synthesis of iron oxide nanoparticles and their catalytic and in vitro anticancer activities. J Cluster Sci 2017;28:245-57.

11. Lv HN, Guo XY, Tu PF, Jiang Y. Comparative analysis of the essential oil composition of Murraya paniculata and M. exotica. Nat Prod Commun 2013;8:1473-5.

12. Latha N, Gowri M. Biosynthesis and characterization of Fe304 nanoparticles using Carica papaya leaves extract. Int J Sci Res 2014;3:1551-6. 
13. Wang T, Jin X, Chen Z, Megharaj M, Naidu R. Green synthesis of Fe nanoparticles using eucalyptus leaf extracts for treatment of eutrophic wastewater. Sci Total Environ 2014;466:210-3.
14. Wu W, He Q Jiang C. Magnetic iron oxide nanoparticles: synthesis and surface functionalization strategies. Nanoscale Res Lett 2008;3:397. 